\title{
ON THE PERFORMANCE OF ROUGH INCLINED STEPPED COMPOSITE BEARINGS WITH MICROPOLAR FLUID
}

\author{
Neminath Bhujappa Naduvinamani \\ Department of Mathematics, Gulbarga University, Gulbarga-585107, INDIA, naduvinamaninb@yahoo.co.in \\ Siddangouda Apparao \\ Department of Mathematics, Appa Institute of Engineering \& Technology, Gulbarga-585104, INDIA.
}

Follow this and additional works at: https://jmstt.ntou.edu.tw/journal

Part of the Engineering Commons

\author{
Recommended Citation \\ Naduvinamani, Neminath Bhujappa and Apparao, Siddangouda (2010) "ON THE PERFORMANCE OF ROUGH \\ INCLINED STEPPED COMPOSITE BEARINGS WITH MICROPOLAR FLUID," Journal of Marine Science and Technology. \\ Vol. 18: Iss. 2, Article 9. \\ DOI: $10.51400 / 2709-6998.2322$ \\ Available at: https://jmstt.ntou.edu.tw/journal/vol18/iss2/9 \\ This Research Article is brought to you for free and open access by Journal of Marine Science and Technology. It has been \\ accepted for inclusion in Journal of Marine Science and Technology by an authorized editor of Journal of Marine Science and \\ Technology.
}


ON THE PERFORMANCE OF ROUGH INCLINED STEPPED COMPOSITE BEARINGS WITH MICROPOLAR FLUID

\section{Acknowledgements}

The authors thankful to the referee for his valuable com- ments on the earlier draft of the paper. The authors sincerely acknowledge the financial assistance by the University Grants Commission, New Delhi, INDIA under the major research project No. F.31-84/2005(SR). 


\title{
ON THE PERFORMANCE OF ROUGH INCLINED STEPPED COMPOSITE BEARINGS WITH MICROPOLAR FLUID
}

\author{
Neminath Bhujappa Naduvinamani* and Siddangouda Apparao**
}

Key words: inclined stepped composite bearing, surface roughness, micropolar fluid.

\begin{abstract}
In this paper, a theoretical analysis of the effect of surface roughness on the lubrication characteristics of inclined stepped composite bearings with micropolar fluid is examined A generalized form of surface roughness is mathematically modeled by a stochastic random variable with non-zero mean, variance and skewness. The generalized average Reynolds type equation is derived for the rough inclined stepped composite bearings with micropolar fluid. The closed form expressions are obtained for the fluid film pressure, load carrying capacity, frictional force. These expressions can be utilized to obtain the performance characteristics of four different types of bearing system viz; rough plane inclined slider, rough composite tapered land bearing, rough stepped bearing and rough composite tapered concave bearing. The numerical computations of the results show that the negatively skewed surface roughness pattern increases fluid film pressure, load carrying capacity and decreases the coefficient of friction whereas adverse effects were found for the positively skewed surface roughness pattern. Further, the rough inclined stepped slider bearing provides the largest load carrying capacity and the least coefficient of friction as compared to the other three geometries under consideration.
\end{abstract}

\section{INTRODUCTION}

In recent years, a considerable amount of tribology research has been devoted to the study of the effect of surface roughness or geometric imperfections on hydrodynamic lubrication, mainly because, in practice, most of the bearing surfaces are rough. The aspect ratio and the absolute height of the asper-

Paper submitted 10/03/08; revised 05/12/09; accepted 05/30/09. Author for correspondence: Neminath Bhujappa Naduvinamani (e-mail: naduvinamaninb @yahoo.co.in).

*Department of Mathematics, Gulbarga University, Gulbarga-585106, INDIA.

**Department of Mathematics, Appa Institute of Engineering \& Technology, Gulbarga-585103, INDIA. ities and valleys observed under microscope vary greatly, depending on material properties and on the method of surface preparation. The surface roughness height may range from $0.05 \mu \mathrm{m}$ or less on polished surfaces to $10 \mu \mathrm{m}$ on medium surfaces. In general, the height of roughness asperities is of the same order as the mean separation in a lubricated contact.

Several approaches have been proposed to study the effect of surface roughness on the performance characteristics of hydrodynamic bearings. Davies [5] employed a saw-tooth curve to mathematically model the surface roughness. The introduction of the stochastic concept to lubrication of rough bearing problems by Tzeng and Saibel [16] has fascinated many researchers and stimulated a fair amount of work in this field. Christensen and Tonder [2] used the stochastic concept to analyze the influence of transverse and longitudinal roughness on the steady state behavior of journal bearings. While Elrod [6] employed the perturbation technique to study the surface roughness effects. Recently Naduvinamani et al. [11] have studied the effect of surface roughness on hydrodynamic lubrication of slider bearings with couplestress fluids by assuming a generalized form of surface roughness characterized by a stochastic random variable with non-zero mean, variance and skewness. The lubrication behavior of different Newtonian and non-Newtonian fluids have been examined and analyzed by many researchers. It is observed that in many appliances of modern industrial lubrication technology, the use of non-Newtonian fluids as lubricants is more important than that of Newtonian fluids. The experimental results reviewed that the lubricants containing polymer additives exhibit enhanced load carrying capacity [9]. The additives minimize the sensitivity of lubricant to the changes in shear rate and thus increase the load carrying capacity of the lubricant. The large sized polymer molecules from the microstructures within the lubricant performing individual motions supporting stress and body moments. Such fluids with microstructures can be adequately represented by micropolar fluid model due to Eringen [7, 8].

Several investigators used the micropolar fluid theory for the study of several bearing systems. The generalized Reynolds equation for micropolar lubricants is derived by Shukla and Isa [15]. They found that the maximum load carrying capacity and corresponding frictional force increases with increase of the parameter characterizing the microstructure 


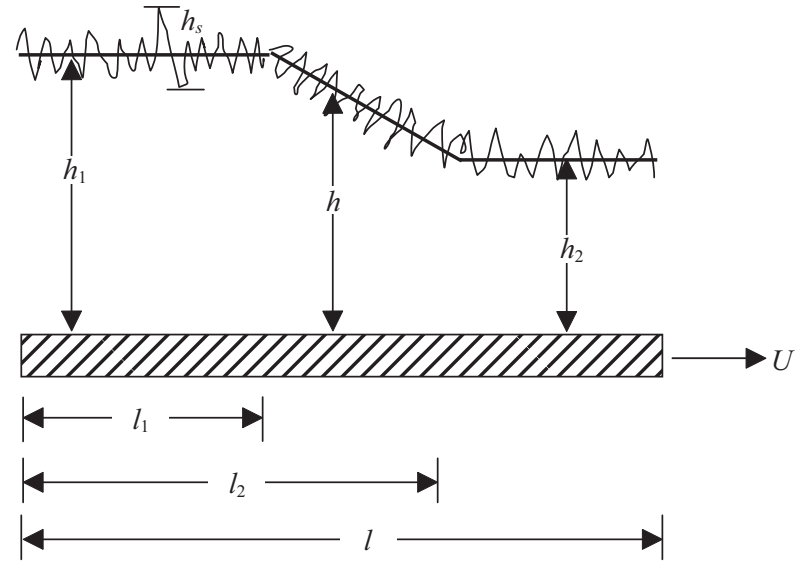

(a)

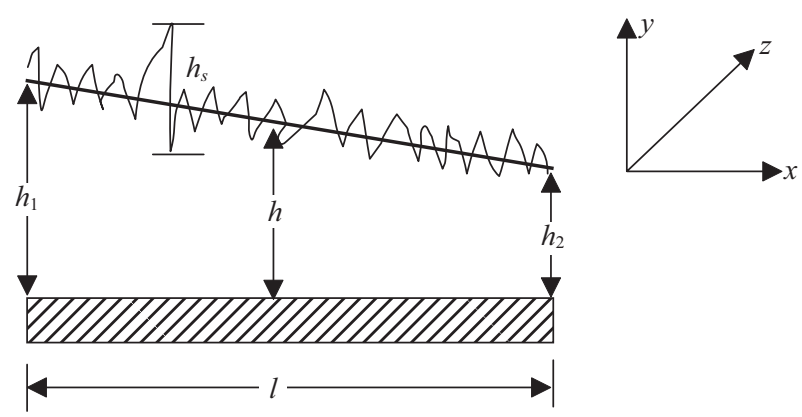

(b)

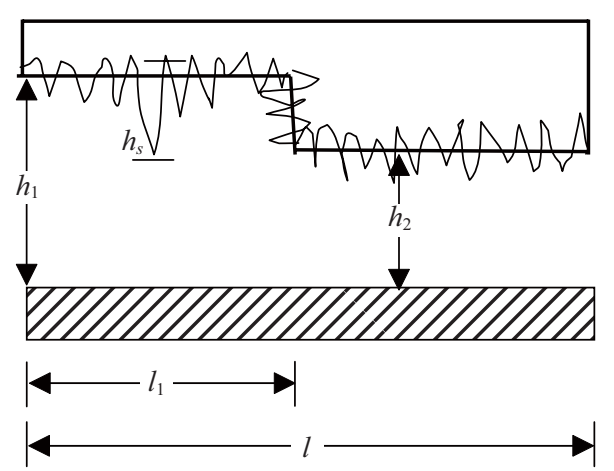

(d)

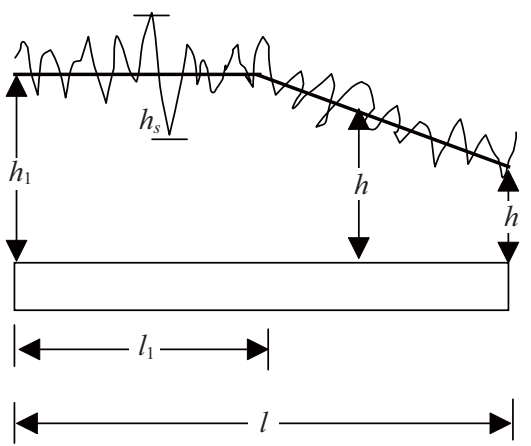

(e)

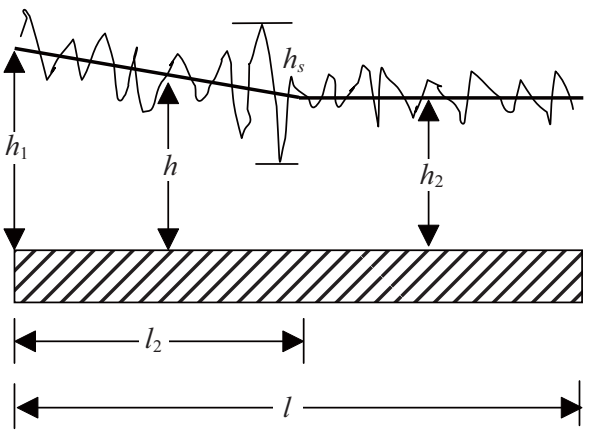

(c)

Fig. 1. (a) Rough inclined stepped composite bearing, (b) rough plane slider bearing, (c) rough composite tapered land bearing, (d) rough stepped bearing, and (e) rough composite tapered concave bearing.

imparted to the lubricant by the presence of additive. The squeeze film and externally pressurized bearings lubricated with micropolar fluid is studied by Agrwal et al. [1]. The dynamically loaded micropolar fluid squeeze film lubrication in journal bearing under fluctuating loads is studied by Prawal Sinha [13]. Maiti [10] studied the composite and step slider bearings in micropolar fluid. The effect of additives in the lubricant of a composite bearing with an inclined stepped surface is presented by Sinha and Singh [14]. The dynamic Reynolds equation for micropolar fluid lubrication of plane inclined slider bearings with squeezing effect is analyzed by Naduvinamani et al. [12]. Das et al. [3, 4] presented the sta- bility characteristics of hydrodynamic journal bearings lubricated with micropolar fluids.

In this paper, an attempt has been made to study the effect of surface roughness on the lubrication characteristics of inclined stepped composite bearings with micropolar fluid by considering the generalized form of surface roughness characterized by a stochastic random variable with non-zero mean, variance and skewness.

\section{DERIVATION}

The geometry under consideration is shown in Fig. 1(a). 
The bearing configuration consists of two surfaces separated by a lubricant film. The $x$-axis is taken along the lower plane across its length, while the $y$-axis is taken across the lubricant film. The lower surface is moving with a velocity $U$ in its own plane.

To represent the surface roughness, the mathematical expression for film thickness is considered to be consisting of two parts

$$
H(x)=h(x)+h_{s}
$$

where $h(x)$ is the mean film thickness and $h_{s}$ is a randomly varying quantity measured from the mean level and thus characterizes the surface roughness. Further, stochastic part $h_{s}$ is considered to have the probability density function $f\left(h_{s}\right)$ defined over the domain $-c \leq h_{s} \leq c$, where $c$ is the maximum deviation from the mean film thickness.

With reference to the Fig. 1(a) the film thickness, $h(x)$, is defined by

$$
h(x)=\left\{\begin{array}{lrl}
h_{1} & \text { for } & 0 \leq x \leq l_{1} \\
h_{1}+\frac{h_{1}-h_{2}}{l_{2}-l_{1}}\left(l_{1}-x\right) & \text { for } & l_{1} \leq x \leq l_{2} \\
h_{2} & \text { for } & l_{2} \leq x \leq 1 .
\end{array}\right.
$$

where $h_{1}$ is the inlet film thickness and $h_{2}$ is the outlet film thickness.

The mean $\alpha^{*}$, the standard deviation $\sigma^{*}$ and the parameter $\varepsilon^{*}$, which is the measure of symmetry of the random variable $h_{s}$, are defined as

$$
\begin{gathered}
\alpha^{*}=E\left(h_{s}\right), \\
\sigma^{*^{2}}=E\left[\left(h_{s}-\alpha^{*}\right)^{2}\right], \\
\varepsilon^{*}=E\left[\left(h_{s}-\alpha^{*}\right)^{3}\right],
\end{gathered}
$$

where $E$ is an expectancy operator defined by

$$
E(\cdot)=\int_{-\infty}^{\infty}(\cdot) f\left(h_{s}\right) d h_{s}
$$

where the parameters $\alpha^{*}, \sigma^{*}$ and $\varepsilon^{*}$ are all independent of $x$. The mean $\alpha^{*}$ and the parameter $\varepsilon^{*}$ can assume both positive and negative values, whereas $\sigma^{*}$ always assumes positive values. The particular case of $\varepsilon^{*}=0$ refers to the symmetric distribution, $\varepsilon^{*}<0$ refers to the negatively skewed surface roughness and $\varepsilon^{*}>0$ refers to the positively skewed surface roughness.

The lubricant in the film region is assumed to be micropolar fluid. It is also assumed that, the body forces and body couples are absent and the characteristic coefficients across the film of the micropolar fluid are constant. Taking the velocity vector $\vec{V}=(u, v, 0)$ and the microrotation vector $\vec{v}=(0,0, v)$, the equations of motion of the fluid reduce to

$$
\begin{gathered}
\rho \frac{D u}{D t}=-\frac{\partial p}{\partial x}+\left(\mu+\frac{\chi}{2}\right)\left(\frac{\partial^{2} u}{\partial x^{2}}+\frac{\partial^{2} u}{\partial y^{2}}\right)+\chi \frac{\partial v_{3}}{\partial y}, \\
\rho \frac{D v}{D t}=-\frac{\partial p}{\partial y}+\left(\mu+\frac{\chi}{2}\right)\left(\frac{\partial^{2} v}{\partial x^{2}}+\frac{\partial^{2} v}{\partial y^{2}}\right)-\chi \frac{\partial v_{3}}{\partial x}, \\
\rho j \frac{D v_{3}}{D t}=\chi\left(\frac{\partial v_{3}}{\partial x}-\frac{\partial u}{\partial y}\right)-2 \chi v_{3}+\gamma\left(\frac{\partial^{2} v_{3}}{\partial x^{2}}+\frac{\partial^{2} v_{3}}{\partial y^{2}}\right), \\
\frac{\partial u}{\partial x}+\frac{\partial v}{\partial y}=0 .
\end{gathered}
$$

Where $\rho$ is the density, $p$ is the pressure and $\mu$ is the shear viscosity, $\chi$ and $\gamma$ are the new material constants peculiar to micropolar fluids, $j$ is the moment of micro inertia and $D / D t$ is the material derivative.

The following usual assumptions of hydrodynamic lubrication of thin films [17] are made in the derivation of modified Reynolds equation:

1. The fluid film thickness $h$ is very much small than the length of the bearing.

2. The inertia terms are negligible in comparison with viscous terms.

3. The variation of pressure across the fluid film is negligible, i.e. $\partial p / \partial y=0$; and

4. The $x$-derivatives of $u$ and $v$ are much smaller than the corresponding $y$ derivatives.

Under these assumptions Eqs. (7)-(10) simplify to

$$
\begin{gathered}
(\mu+\chi / 2) \frac{\partial^{2} u}{\partial y^{2}}+\chi \frac{\partial v_{3}}{\partial y}=\frac{\partial p}{\partial x} \\
\frac{\partial p}{\partial y}=0 \\
\gamma \frac{\partial^{2} v_{3}}{\partial y^{2}}-\chi \frac{\partial u}{\partial y}-2 \chi v_{3}=0 \\
\frac{\partial u}{\partial x}+\frac{\partial v}{\partial y}=0 .
\end{gathered}
$$

The relevant boundary conditions for the velocity and mi- 
cro rotational velocity components are

(i) at the upper surface $(y=H)$

$$
u=0, v_{3}=0, v=0
$$

(ii) at the lower surface $(y=0)$

$$
u=U, v_{3}=0, v=0
$$

The solution of (11) and (13) subject to the boundary conditions (15a) and (15b) are

$$
\begin{aligned}
u= & \frac{1}{\mu} \frac{d p}{d x}\left[\frac{y^{2}}{2}-\frac{N^{2} H}{m} \frac{(\cosh m y-1)}{\sinh m H}\right]+U+ \\
& \frac{D_{1}}{\left(1-N^{2}\right)}\left[y-\frac{N^{2}}{m}\left\{\sinh m y-\frac{(\cosh m y-1)(\cosh m H-1)}{\sinh m H}\right\}\right]
\end{aligned}
$$

$$
\begin{aligned}
v_{3}= & \frac{D_{1}}{2\left(1-N^{2}\right)}(\cosh m y-1) \\
& +\frac{\sinh m y}{\sinh m H}\left[\frac{H}{\mu} \frac{d p}{d x}-\frac{D_{1}}{2\left(1-N^{2}\right)}(\cosh m H-1)\right]-\frac{y}{2 \mu} \frac{d p}{d x}
\end{aligned}
$$

Where

$$
\begin{gathered}
D_{1}=-\frac{\left(1-N^{2}\right)}{2}\left[\frac{H}{\mu} \frac{d p}{d x}+\frac{U}{\left\{\frac{H}{2}-\frac{N^{2}}{m} \frac{(\cosh m H-1)}{\sinh m H}\right\}}\right], \\
m=\frac{N}{L}, N=\left(\frac{\chi}{2 \mu+\chi}\right)^{1 / 2} \text { and } L=\left(\frac{\gamma}{4 \mu}\right)^{1 / 2}
\end{gathered}
$$

The modified Reynolds type equation for the pressure in the film region is obtained by using (16) for $u$ in the continuity equation (14) and then integrating over the film thickness and also using the boundary conditions for $v$ given in (15a) and (15b) as

$$
\frac{d}{d x}\left[\{f(N, L, H)\} \frac{d p}{d x}\right]=6 \mu U \frac{d H}{d x}
$$

where

$$
f(N, L, H)=H^{3}+12 L^{2} H-6 N L H^{2} \operatorname{coth}\left(\frac{N H}{2 L}\right)
$$

Multiplying both sides of (18) by $f\left(h_{s}\right)$ and integrating with respect to $h_{s}$ from $-c$ to $c$ and using (3)-(5), Eq. (18) takes the form

$$
\frac{d}{d x}\left[\left\{\bar{f}\left(N, L, h, \varepsilon^{*}, \alpha^{*}, \sigma^{*}\right)\right\} \frac{d \bar{p}}{d x}\right]=6 \mu U \frac{d h}{d x}
$$

where $E(H)=h ; \bar{p}(=E(p))$ is the expected value of the film pressure $p$,

$$
\begin{gathered}
\bar{f}\left(N, L, h, \varepsilon^{*}, \alpha^{*}, \sigma^{*}\right)=E(f(N, L, H)) \\
=F_{1}-F_{2}\left(F_{3}+F_{4}\right), \\
F_{1}=h^{3}+\varepsilon^{*}+3 \sigma^{*^{2}} \alpha^{*}+3 h^{2} \alpha^{*}+3 h\left(\sigma^{*^{2}}+\alpha^{*^{2}}\right)+\alpha^{*^{3}}+12 L^{2}\left(h+\alpha^{*}\right), \\
F_{2}=6 N L\left(h^{2}+\alpha^{*^{2}}+\sigma^{*^{2}}+2 h \alpha^{*}\right), \\
F_{3}=\left(1-\operatorname{coth}^{2}\left(\frac{N h}{2 L}\right)\right)\left(\frac{N \alpha^{*}}{2 L}-\frac{N^{3}}{24 L^{3}}\left(\varepsilon+\alpha^{*^{3}}+3 \alpha^{*} \sigma^{*^{2}}\right)\right),
\end{gathered}
$$

and

$$
F_{4}=\operatorname{coth}\left(\frac{N h}{2 L}\right)\left(1-\frac{N^{2}}{4 L^{2}}\left(\alpha^{*^{2}}+\sigma^{*^{2}}\right)\right) \text {. }
$$

Once integration of (19) with respect to $x$ gives

$$
\frac{d \bar{p}}{d x}=6 \mu U\left(\frac{h-h_{0}}{\bar{f}\left(N, L, h, \varepsilon^{*}, \alpha^{*}, \sigma^{*}\right)}\right)
$$

where $h_{0}$ is the film thickness at which $\frac{d \bar{p}}{d x}=0$.

Introducing the non-dimensional quantities

$$
\begin{aligned}
& P=\frac{\bar{p} h_{2}^{2}}{\mu U l}, \bar{h}=\frac{h}{h_{2}}, \overline{h_{1}}=\frac{h_{1}}{h_{2}}, \bar{x}=\frac{x}{l}, L^{*}=\frac{L}{h_{2}}, L_{1}=\frac{l_{1}}{l}, \\
& L_{2}=\frac{l_{2}}{l}, \varepsilon=\frac{\varepsilon^{*}}{h_{2}^{3}}, \alpha=\frac{\alpha^{*}}{h_{2}}, \sigma^{2}=\frac{\sigma^{* 2}}{h_{2}^{2}}
\end{aligned}
$$

into (20) one can obtain the nondimensional mean pressure gradient in the form

$$
\frac{d P}{d \bar{x}}=6\left(\frac{\bar{h}-\bar{h}_{0}}{\bar{f}\left(N, L^{*}, \bar{h}, \varepsilon, \alpha, \sigma\right)}\right)
$$

The relevant boundary conditions for the pressure field are 


$$
P=0 \quad \text { at } \quad \bar{x}=0,1
$$

and the pressure is continuous at $\bar{x}=L_{1}$ and $\bar{x}=L_{2}$.

Integration of (21) and the use of boundary conditions (22) gives

$$
\left.\begin{array}{ll}
P=6\left(\frac{\left(\bar{h}_{1}-\bar{h}_{0}\right) \bar{x}}{\bar{f}\left(N, L^{*}, \bar{h}_{1}, \varepsilon, \alpha, \sigma\right)}\right) & 0 \leq \bar{x} \leq L_{1} \\
P=P_{c}+6 \int_{L_{1}}^{\bar{x}}\left(\frac{\bar{h}-\bar{h}_{0}}{\bar{f}\left(N, L^{*}, \bar{h}, \varepsilon, \alpha, \sigma\right)}\right) d \bar{x} & L_{1} \leq \bar{x} \leq L_{2} \\
P=6\left(\frac{\left(1-\bar{h}_{0}\right)(\bar{x}-1)}{\bar{f}\left(N, L^{*}, 1, \mathcal{E}, \alpha, \sigma\right)}\right) & L_{2} \leq \bar{x} \leq 1
\end{array}\right\}
$$

where $P_{c}$ is the dimensionless pressure at $\bar{x}=L_{1}$ and is given by

$$
P_{c}=6\left(\frac{\left(\bar{h}_{1}-\bar{h}_{0}\right) L_{1}}{\bar{f}\left(N, L^{*}, \bar{h}_{1}, \mathcal{\varepsilon}, \alpha, \sigma\right)}\right)
$$

and

$$
\bar{h}_{0}=\frac{A}{B}
$$

where

$$
\begin{aligned}
A= & \frac{\bar{h}_{1} L_{1}}{\bar{f}\left(N, L^{*}, \bar{h}_{1}, \varepsilon, \alpha, \sigma\right)}-\frac{L_{2}-1}{\bar{f}\left(N, L^{*}, 1, \mathcal{E}, \alpha, \sigma\right)} \\
& +\int_{L_{1}}^{L_{2}} \frac{\bar{h} d \bar{x}}{\bar{f}\left(N, L^{*}, \bar{h}, \varepsilon, \alpha, \sigma\right)}
\end{aligned}
$$

and

$$
\begin{aligned}
B= & \frac{L_{1}}{\bar{f}\left(N, L^{*}, \bar{h}_{1}, \varepsilon, \alpha, \sigma\right)}-\frac{L_{2}-1}{\bar{f}\left(N, L^{*}, 1, \varepsilon, \alpha, \sigma\right)} \\
& +\int_{L_{1}}^{L_{2}} \frac{d \bar{x}}{\bar{f}\left(N, L^{*}, \bar{h}, \varepsilon, \alpha, \sigma\right)}
\end{aligned} .
$$

The mean load carrying capacity w per unit width is given by

$$
w=\int_{0}^{l} \bar{p} d x
$$

The non-dimensional load carrying capacity is obtained in the form

$$
\begin{aligned}
W= & \frac{w h_{2}^{2}}{\mu U l^{2}}=\frac{3\left(1-\bar{h}_{0}\right)\left(L_{2}^{2}-1\right)}{\bar{f}\left(N, L^{*}, 1, \mathcal{\varepsilon}, \alpha, \sigma\right)}-3 \frac{\left(\bar{h}_{1}-\bar{h}_{0}\right) L_{1}^{2}}{\bar{f}\left(N, L^{*}, \bar{h}_{1}, \varepsilon, \alpha, \sigma\right)} \\
& +6 \int_{L_{1}}^{L_{2}} \frac{\bar{x}\left(\bar{h}-\bar{h}_{0}\right)}{\bar{f}\left(N, L^{*}, \bar{h}, \varepsilon, \alpha, \sigma\right)} d \bar{x}
\end{aligned}
$$

The frictional force F per unit width on the bearing surface is given by

$$
F=\int_{0}^{l}\left(t_{21}\right)_{y=0} d x
$$

where

$$
\begin{aligned}
& \left(t_{21}\right)_{y=0}=-\frac{H}{2} \frac{d p}{d x}-\frac{\mu U}{g(N, L, H)}, \\
& g(N, L, h)=1-\frac{2 N L}{h} \tanh \left(\frac{N h}{2 L}\right) .
\end{aligned}
$$

The dimensionless frictional force is obtained in the form

$$
\bar{F}=\frac{F h_{2}}{\mu U l}=A_{1}+A_{2}+A_{3}
$$

where

$$
\begin{gathered}
A_{1}=3 \frac{\left(\bar{h}_{1}-\bar{h}_{0}\right) L_{1}}{\bar{f}_{1}\left(N, L^{*}, \bar{h}_{1}, \varepsilon, \alpha, \sigma\right)}+\frac{L_{1}}{G\left(N, L^{*}, \bar{h}_{1}\right)}, \\
A_{2}=\frac{3\left(1-\bar{h}_{0}\right)\left(1-L_{2}\right)}{\overline{f_{1}}\left(N, L^{*}, 1, \varepsilon, \alpha, \sigma\right)}+\frac{\left(1-L_{2}\right)}{G\left(N, L^{*}, 1\right)},
\end{gathered}
$$

$$
\begin{gathered}
A_{3}=\int_{L_{1}}^{L_{2}}\left(3 \frac{\left(\bar{h}-\bar{h}_{0}\right)}{\overline{f_{1}}\left(N, L^{*}, \bar{h}, \varepsilon, \alpha, \sigma\right)}+\frac{1}{G\left(N, L^{*}, \bar{h}\right)}\right) d \bar{x}, \\
\overline{f_{1}}\left(N, L, h, \varepsilon^{*}, \alpha^{*}, \sigma^{*}\right)=I_{1}-I_{2}\left(I_{3}+I_{4}\right), \\
I_{1}=\bar{h}^{2}+\alpha^{2}+\sigma^{2}+2 \bar{h} \alpha+12 L^{*^{2}}, \\
I_{2}=6 N L^{*}(\bar{h}+\alpha),
\end{gathered}
$$

$$
I_{3}=\left(1-\operatorname{coth}^{2}\left(\frac{N \bar{h}}{2 L^{*}}\right)\right)\left(\frac{N \alpha}{2 L^{*}}-\frac{N^{3}}{24 L^{*^{3}}}\left(\varepsilon+\alpha^{3}+3 \alpha \sigma^{2}\right)\right) \text {, }
$$




$$
\begin{gathered}
I_{4}=\operatorname{coth}\left(\frac{N \bar{h}}{2 L^{*}}\right)\left(1-\frac{N^{2}}{4 L^{*^{2}}}\left(\alpha^{2}+\sigma^{2}\right)\right) \\
G\left(N, L^{*}, \bar{h}\right)=g_{1}-g_{2}\left(g_{3}+g_{4}\right), \\
g_{1}=(\bar{h}+\alpha), g_{2}=2 N L^{*}, g_{3}=\tanh \left(\frac{N \bar{h}}{2 L^{*}}\right) \text { and } \\
g_{4}=\left(1-\tanh ^{2}\left(\frac{N \bar{h}}{2 L^{*}}\right)\right)\left(\frac{N \alpha}{2 L^{*}}-\frac{N^{3}}{24 L^{*^{3}}}\left(\varepsilon+\alpha^{3}+3 \sigma^{2} \alpha\right)\right)
\end{gathered}
$$

The coefficient of friction is given by

$$
C=\frac{\bar{F}}{W}
$$

\section{PARTICULAR CASES}

Equations (23), (25) and (27) can be used respectively to determine the pressure distribution, load carrying capacity and frictional force for the following cases:

1. The rough plane inclined bearing (Fig. 1(b)) when $L_{1}=0$ and $L_{2}=1$.

2. The rough composite tapered land bearing (Fig. 1(c)) when $L_{1}=0$.

3. The rough stepped bearing (Fig. 1(d)) when $L_{1}=L_{2}$.

4. The rough composite tapered concave bearing (Fig. 1(e)) when $L_{1}=1$.

\section{RESULTS AND DISCUSSION}

This paper predicts the effect of surface roughness on the performance characteristics of inclined stepped composite bearing lubricated with micropolar fluid. The micropolar fluid is characterized by two dimensionless parameters $N$ and $L^{*}$. The coupling number $N$ characterizes the coupling of the linear and rotational motion arising from the micromotion of the fluid molecules or the additive molecules. Thus the coupling number $N(0 \leq N \leq L)$ signifies the coupling between the Newtonian and rotational viscosity. As $\chi$ tends to zero, $N$ also tends to zero, and the expressions for the bearing characteristics reduce to their counterparts in Newtonian theory.

The parameter $L$ has the dimensions of length and can be identified with some property which depends on the size of the molecules, say the chain length of the polar additive molecule in a non-polar lubricant. Thus $L$ can be considered as a characterization of the interaction of the fluid with the bearing geometry. Micropolar effects are expected to be important either when the characteristic material length is small, i.e. the additive has a long chain length, or when the clearance width $h_{2}$ is large. As $L^{*} \rightarrow 0$ the expressions for the bearing char-

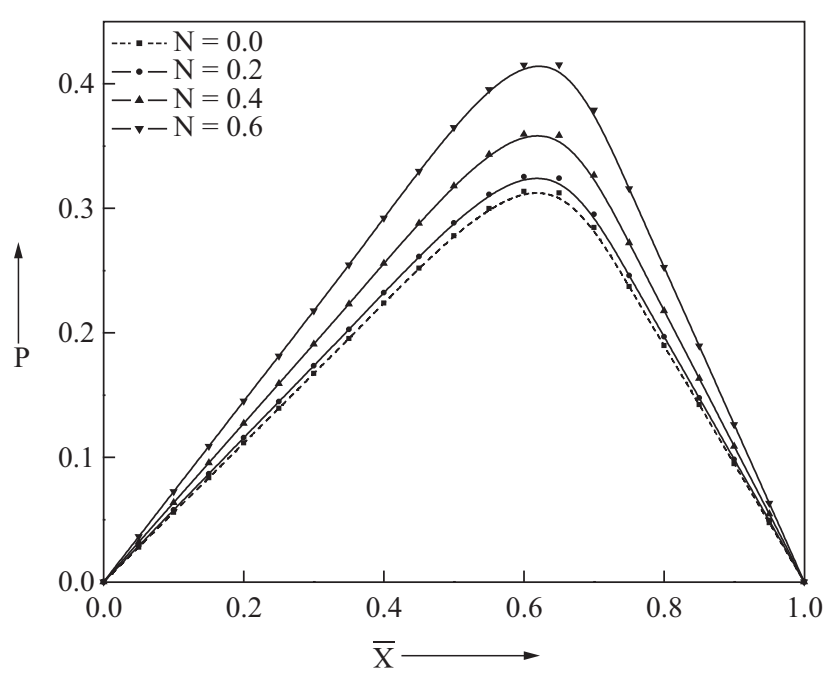

Fig. 2. Variation of nondimensional pressure $P$ with dimensionless $\mathbf{x}$ coordinate for different values of $N$ with $L^{*}=0.2, \alpha=0.05, \varepsilon=0.05$, $\sigma=\mathbf{0 . 1}$.

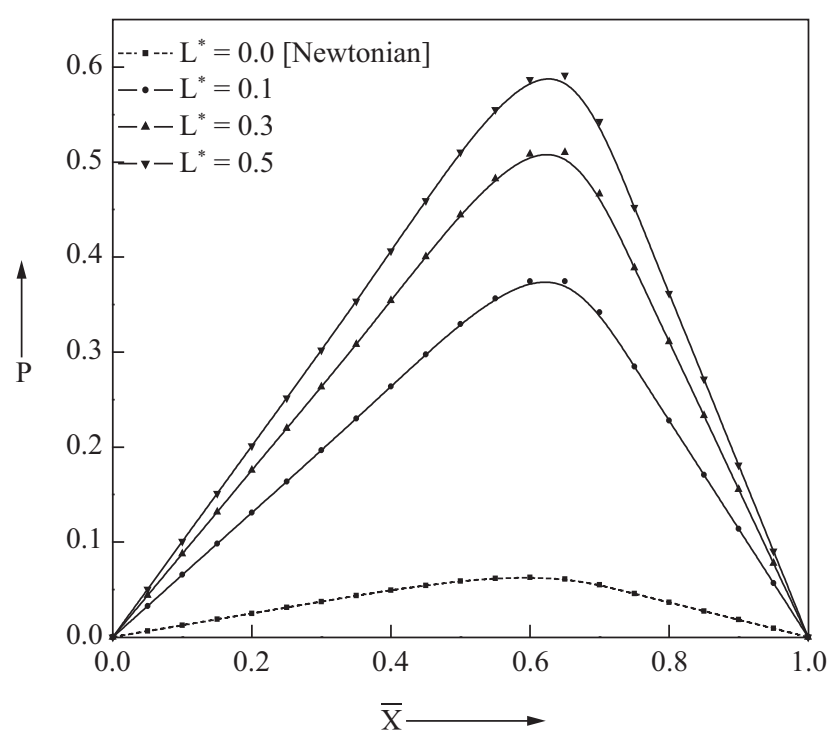

Fig. 3. Variation of nondimensional pressure $P$ with dimensionless $x$ coordinate for different values of $L^{*}$ with $N=0.7, \alpha=0.05, \varepsilon=0.05$, $\sigma=0.1$.

acteristics reduce to their equivalents in Newtonian theory with $\mu$ replaced every where by $\left(\mu+\frac{1}{2} \chi\right)$.

The effect of surface roughness characterized by the parameter $\alpha, \sigma$ and $\varepsilon$. The range of values for $\alpha$ and $\varepsilon$ are so chosen that, the coressoponding hydrodynamic film shapes are feasible. Bearing characteristics were obtained for the rough inclined stepped bearing with $L_{1}=0.3, L_{2}=0.7$ and $\bar{h}_{1}=2.0$.

\section{PRESSURE DISTRIBUTION}

The effect of micropolarity on the variations of non- 


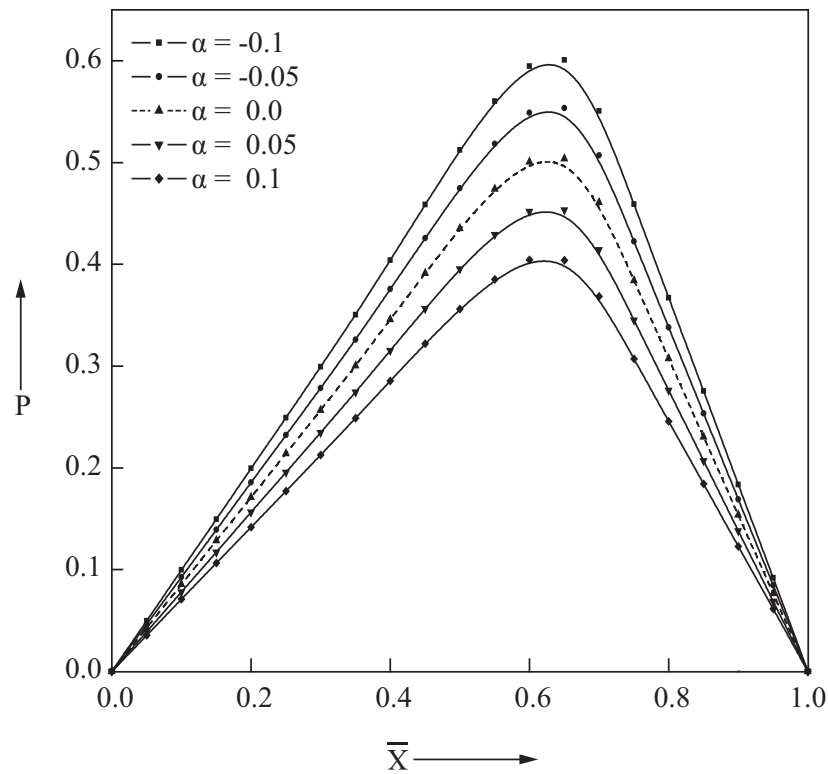

Fig. 4. Variation of nondimensional pressure $P$ with dimensionless $x$ coordinate for different values of $\alpha$ with $N=0.7, L^{*}=0.2, \varepsilon=0.05$, $\sigma=0.1$.

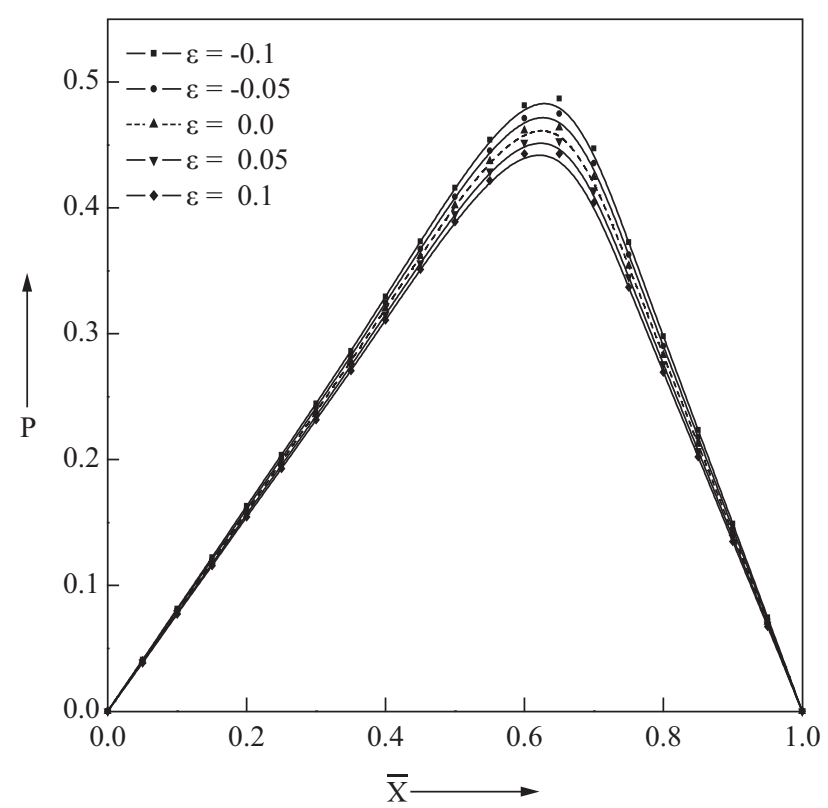

Fig. 5. Variation of nondimensional pressure $P$ with dimensionless $x$ coordinate for different values of $\varepsilon$ with $N=0.7, L^{*}=0.2, \alpha=0.05$, $\sigma=0.1$.

dimensional mean film pressure $P$ with $\bar{x}$ for different values of $N$ and $L^{*}$ is depicted in the Figs. 2 and 3. It is observed that $P$ increases for increasing values of $N$ and $L^{*}$ as compared to the corresponding Newtonian case. The effect of roughness parameters $\alpha$ and $\varepsilon$ on the variations of non-dimensional pressure $P$ with $\bar{x}$ is shown in Figs. 4-5.

It is observed that $P$ increases for negatively increasing values of $\alpha$ and $\varepsilon$ whereas reverse trend is observed for posi-

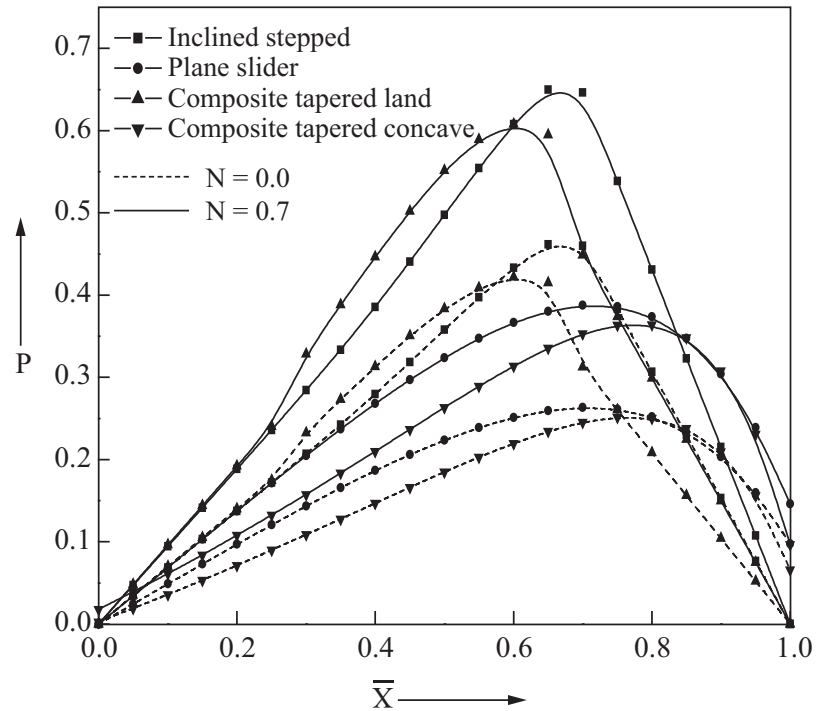

Fig. 6. Variation of nondimensional pressure $P$ with dimensionless $x$ coordinate for different types of bearings with $L^{*}=0.2, \alpha=0.05, \varepsilon$ $=0.05, \sigma=0.1$.

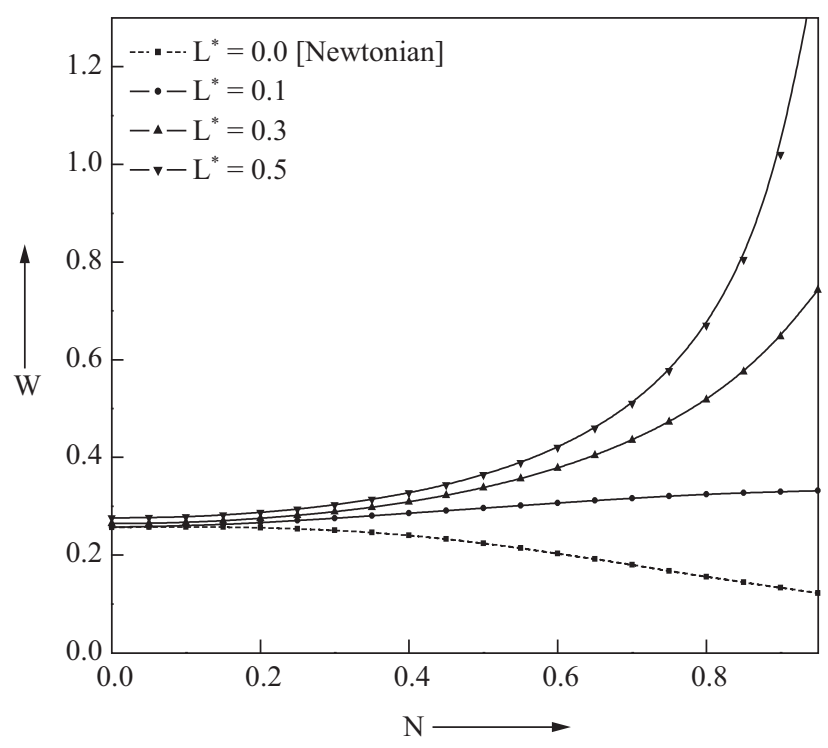

Fig. 7. Variation of dimensionless load $W$ with $N$ for different values of $L^{*}$ with $\alpha=0.05, \varepsilon=0.05, \sigma=0.1$.

tively increasing values of $\alpha$ and $\varepsilon$. The effect of coupling number $N$ on the variations of $P$ with $\bar{x}$ is presented in the Fig. 6 for different bearing geometries. It is observed that, the rough inclined stepped bearing generates more pressure as compared to all other bearing geometries.

\section{LOAD CARRYING CAPACITY}

The variation of dimensionless load carrying capacity $W$ with $N$ for different values of $L^{*}$ is depicted in the Fig. 7 .

Compared with the Newtonian lubricant case the effect of 


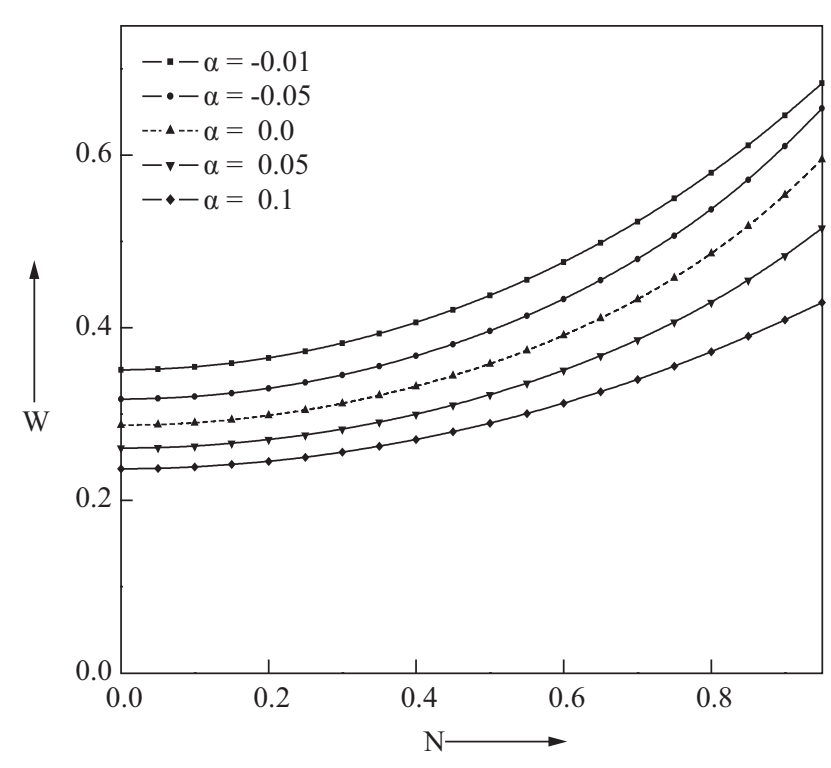

Fig. 8. Variation of dimensionless load $W$ with $N$ for different values of $\alpha$ with $L^{*}=0.2, \varepsilon=0.05, \sigma=0.1$.

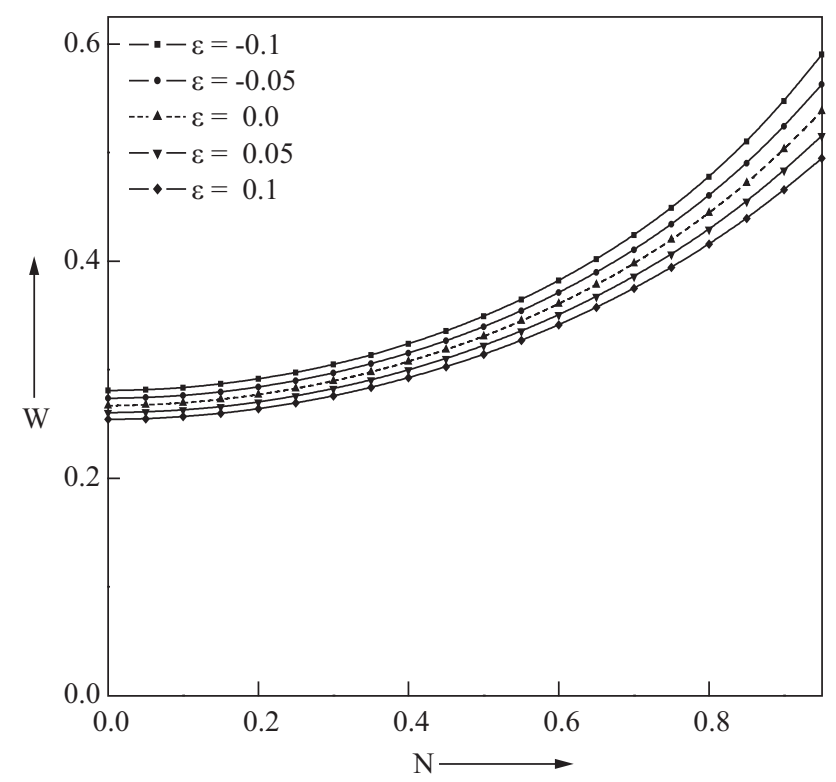

Fig. 9. Variation of dimensionless load $W$ with $N$ for different values of $\varepsilon$ with $L^{*}=0.2, \alpha=0.05, \sigma=0.1$.

micropolar fluid increases the load carrying capacity for increasing values of $L^{*}$. Figures 8-9 depicts the variations of dimensionless load carrying capacity $W$ with $N$ for different values of surface roughness parameters $\alpha$ and $\varepsilon$ respectively. It is observed that, the negatively increasing values of $\alpha$ and $\varepsilon$ increases the load carrying capacity and positively increasing values of $\alpha$ and $\varepsilon$ decreases the load carrying capacity.

The variation of load carrying capacity, $W$ with $N$ is depicted in the Fig. 10 for different values of $L^{*}$ for all the bearing geometries under consideration. It is observed that the load carrying capacity of the rough inclined stepped slider

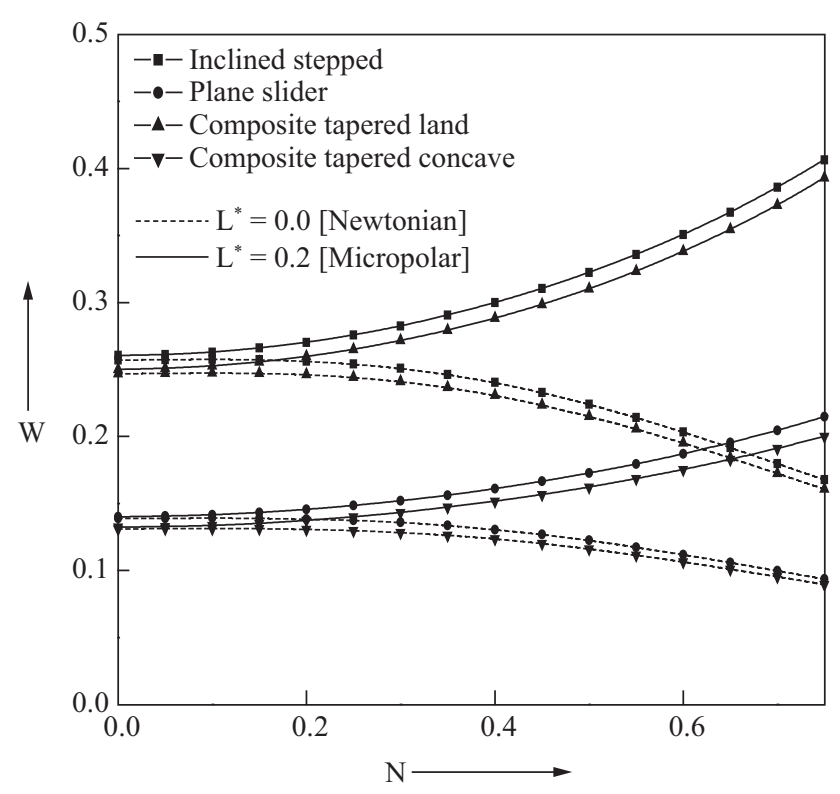

Fig. 10. Variation of dimensionless load $W$ with $N$ for different types of bearings with $\alpha=0.05, \varepsilon=0.05, \sigma=0.1$.

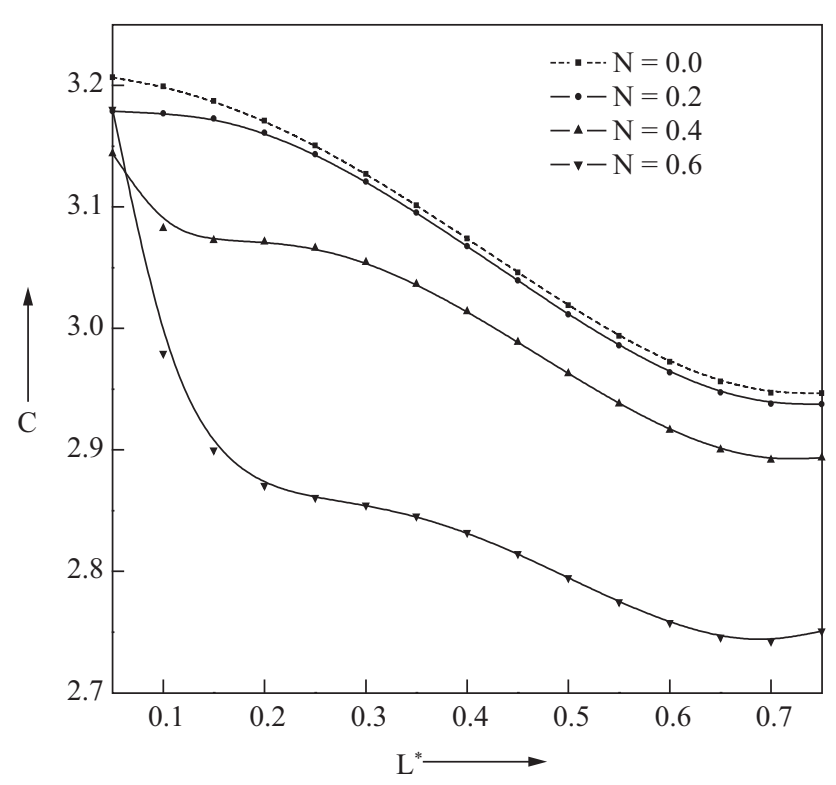

Fig. 11. Variation of coefficient of friction $C$ with $L^{*}$ for different values of $N$ with $\alpha=0.05, \varepsilon=0.05, \sigma=0.1$.

bearing is larger as compared to all other geometries under consideration.

\section{COEFFICIENT OF FRICTION}

Figure 11 shows the effect of micropolar fluid on the variations of the coefficient of friction with $L^{*}$ for different values of $N$.

The most interesting aspect of these curves is the fact that the coefficient of friction tends to decrease as $N$ increases. The effect of roughness parameters $\alpha$ and $\varepsilon$ on the variation of $C$ 


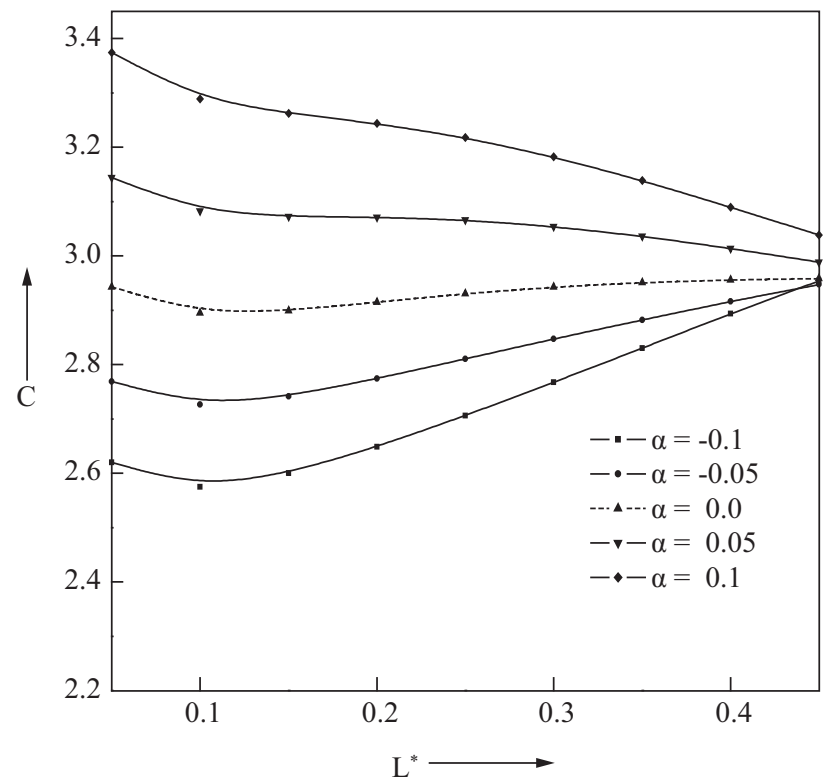

Fig. 12. Variation of coefficient of friction $C$ with $L^{*}$ for different values of $\alpha$ with $N=0.4, \varepsilon=0.05, \sigma=0.1$.

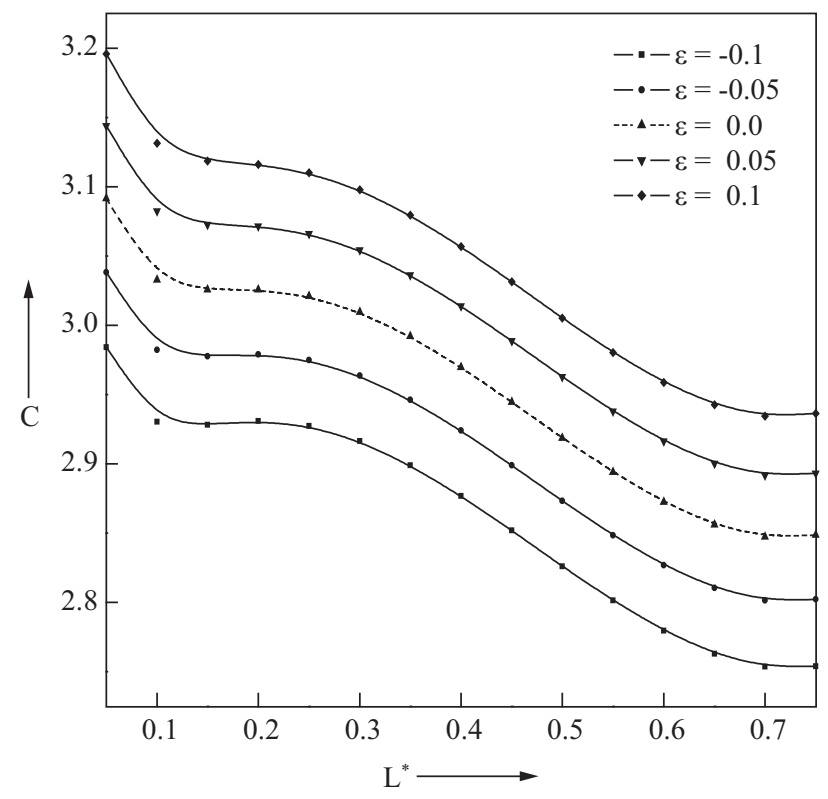

Fig. 13. Variation of coefficient of friction $C$ with $L^{*}$ for different values of $\varepsilon$ with $N=0.4, \alpha=0.05, \sigma=0.1$.

with $L^{*}$ is depicted in Figs. 12-13.

It is observed that negatively increasing values of $\alpha$ and $\varepsilon$ decreases $C$ whereas reverse trend is observed for the positively increasing values of $\alpha$ and $\varepsilon$.

The variation of coefficient of friction $C$ with $L^{*}$ is depicted in Fig. 14 for different values of $N$ for all the bearing geometries under consideration. It is observed that, the rough composite tapered concave slider bearing results in a least coefficient of friction as compared to all other geometries.

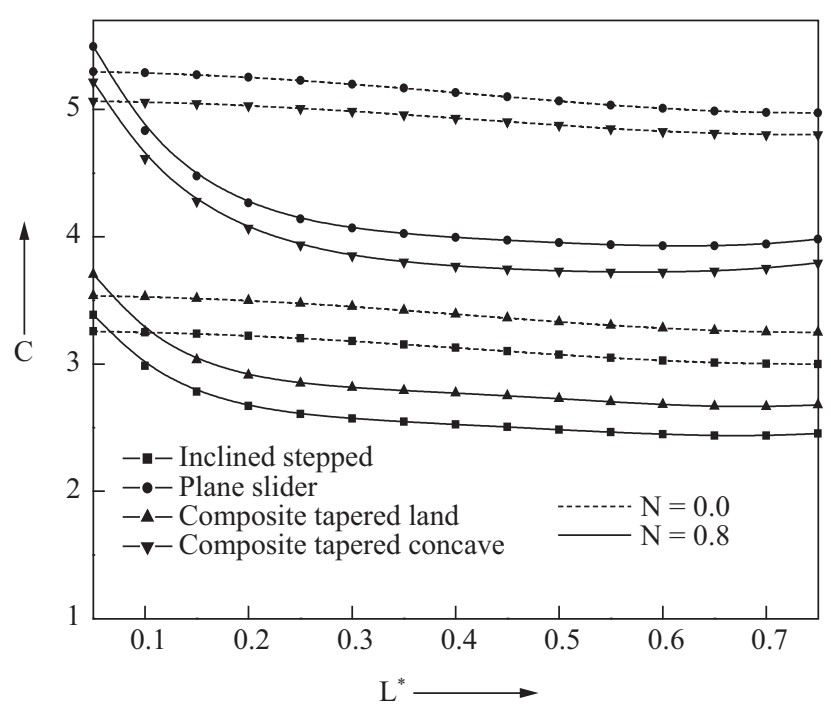

Fig. 14. Variation of coefficient of friction $C$ with $L^{*}$ for different types of bearings with $\alpha=0.05, \varepsilon=0.05, \sigma=0.1$.

\section{CONCLUSIONS}

On the basis of Eringen's [7] micropolar fluid theory and general stochastic method for the study of surface roughness, this paper predicts the effect of surface roughness on the inclined stepped composite bearing with micropolar fluid. On the basis of numerical computations of the results obtained, the following conclusions are drawn.

1. The presence of microstructure additives in the lubricant provides an enhancement in the fluid film pressure and the load carrying capacity and decreases the coefficient of friction as compared to the corresponding Newtonian case.

2. The negatively skewed surface roughness increases the fluid film pressure, load carrying capacity and decreases the coefficient of friction.

3. The performance of the bearing suffers on account of positively skewed surface roughness pattern.

4. The rough inclined stepped composite bearing has the largest load carrying capacity and the smallest coefficient of friction as compared to the rough plane slider and rough composite tapered land slider bearings.

\section{ACKNOWLEDGMENTS}

The authors thankful to the referee for his valuable comments on the earlier draft of the paper. The authors sincerely acknowledge the financial assistance by the University Grants Commission, New Delhi, INDIA under the major research project No. F.31-84/2005(SR). 


\section{NOMENCLATURE}

C coefficient of friction

c maximum deviation from the mean film thickness

E expectancy operator

$F \quad$ frictional force

$\bar{F} \quad$ non-dimensional frictional force $\left(=\frac{F h_{2}}{\mu U l}\right)$

$H(x) \quad$ film thickness $\left(=h(x)+h_{s}\right)$

$h(x) \quad$ mean film thickness

$h_{1} \quad$ inlet film thickness

$h_{0}(x) \quad$ film thickness when $(d p / d x)=0$

$\bar{h}_{1}(x)$ dimensionless inlet film thickness $\left(=h_{1} / h_{2}\right)$

$h_{2} \quad$ minimum film thickness

$\bar{h}_{0}(x)$ dimensionless film thickness when $(d p / d x)=0$

$h_{s} \quad$ stochastic film thickness measured from the nominal mean levels of the bearing surfaces

$L \quad$ material length, $(\gamma / 4 \mu)^{1 / 2}$

$L_{1} \quad$ non-dimensional length of leading parallel portion $(=1 / l)$

$L_{2} \quad$ sum of the non-dimensional length of the leading parallel portion and the inclined portion $\left(=l_{2} / l\right)$ total length of the sliding surface along the direction of motion

$l_{1} \quad$ length of leading parallel portion

$l_{2} \quad$ sum of the length of the leading parallel portion and the inclined portion

$N \quad$ coupling number, $(\chi / 2 \mu+\chi)^{1 / 2}$

$p \quad$ lubricant film pressure

$\bar{p} \quad$ expected value of the lubricant film pressure $(=E(p))$

$P \quad$ dimensionless film pressure $\left(=\frac{\bar{p} h_{2}^{2}}{\mu U l}\right)$

$w \quad$ load carrying capacity

$W \quad$ non-dimensional load carrying capacity $\left(=\frac{w h_{2}^{2}}{\mu U l^{2}}\right)$

$x, y \quad$ cartesian coordinates

$\bar{x} \quad$ dimensionless form of $\mathrm{x}(=x / l)$

$\alpha^{*} \quad$ mean of the stochastic film thickness

$\sigma^{*} \quad$ standard deviation of the film thickness

$\varepsilon^{*} \quad$ measure of symmetry of the stochastic random variable

$\alpha$

non-dimensional form of $\alpha^{*}\left(=\alpha^{*} / h_{2}\right)$ $\varepsilon \quad$ non-dimensional form of $\varepsilon^{*}\left(=\varepsilon^{*} / h_{2}^{3}\right)$

$\sigma \quad$ non-dimensional form of $\sigma^{*}\left(=\sigma^{*} / h_{2}\right)$

$\gamma, \chi \quad$ viscosity coefficients for micropolar fluids

$\mu \quad$ classical viscosity coefficient

\section{REFERENCES}

1. Agrwal, V. K., Ganju, K. L., and Jethi, S. C., "Squeeze film and externally pressurized bearings with micropolar fluids," Wear, Vol. 19, pp. 259-265 (1972).

2. Christensen, H. and Tonder, K., "The hydrodynamic lubrication of rough journal bearings," ASME Journal of Lubrication Technology, pp. 166-171 (1973).

3. Das, S., Guha, S. K., and Chattopadhyay, A. K., "Linear stability analysis of hydrodynamic journal bearings under micropolar lubrication," Tribology International, Vol. 38, pp. 500-507 (2005).

4. Das, S., Guha, S. K., and Chattopadhyay, A. K., "On the conical whirl instability of hydrodynamic journal bearings lubricated with micropolar fluids," Proceedings of Institution of Mechanical Engineers, Part J: Journal of Engineering Tribology, Vol. 215, pp. 431-439 (2001).

5. Davies, M. G., "The generation of pressure between rough fluid lubricated, moving deformable surfaces," Lubrication Engineering, Vol. 19, pp. 246 (1963).

6. Elrod, H. G., A General Theory for Laminar Lubrication with Reynolds Roughness, Report for Department of Machine Design, Technical University of Denmark (1977).

7. Eringen, A. C., "Theory of micropolar fluids," Journal of Mathematics and Mechanics, Vol. 16, pp. 1-18 (1966).

8. Eringen, A. C., "Simple microfluids," International Journal of Engineering Science, Vol. 2, pp. 205-217 (1964).

9. Larson, C. M. and Larson, R., "Lubricant additives," in: O'Connor, J. J. and Boyd, J. (eds.), Standard Handbook of Lubrication Engineering, McGraw Hill, New York (1968).

10. Maiti, G., "Composite and step slider bearings in micropolar fluid," Japanese Journal of Applied Physics, Vol. 12, No. 7, pp. 1058-1064 (1973).

11. Naduvinamani, N. B., Fathima, S. T., and Hiremath, P. S., "Hydrodynamic lubrication of rough slider bearing with couple stress fluids," Tribology International, Vol. 36, pp. 949-959 (2003).

12. Naduvinamani, N. B. and Marali, G. B., "Dynamic Reynolds equation for micropolar fluids and the analysis of plane inclined slider bearings with squeezing effect," Proceedings of Institution of Mechanical Engineers, Part J: Journal of Engineering Tribology, Vol. 215, No. J7, pp. 823-829 (2007).

13. Prawal S., "Dynamically loaded micropolar fluid lubricated journal bearings with special reference to squeeze films under fluctuating loads," Wear, Vol. 45, pp. 279-292 (1977).

14. Prawal, S. and Chandan, S. "The effect of additives in the lubricant of a composite bearing with an inclined stepped surface," Wear, Vol. 66, pp. 17-26 (1981).

15. Shukla, J. B. and Isa, M., "Generalized Reynolds equation for micropolar lubricants and its application to one dimensional slider bearings: effects of solid particle additives in solution," Journal of Mechanical Engineering Science, Vol. 17, No. 5, pp. 280-284 (1975).

16. Tzeng, S. T. and Saibel, E., "Surface roughness effect on slider bearing lubrication," ASLE Transactions, Vol. 10, pp. 334 (1967).

17. Zaheeruddin, K. H. and Isa, M., "Micropolar fluid lubrication of one-dimensional journal bearing," Wear, Vol. 50, pp. 211-220 (1978). 